\title{
Pulmonary lymphangioleiomyomatosis in a 46-year-old female: A case report and review of the literature
}

\author{
BO ZHOU*, QINYUE GUO*, HONG ZHOU, WANKE XIE, \\ TAO XUE, MANXIANG LI, LAN YANG and ZHIHONG SHI \\ Department of Respiratory and Critical Care Medicine, The First Affiliated \\ Hospital of Xi'an Jiaotong University, Xi'an, Shaanxi 710061, P.R. China
}

Received January 20, 2016; Accepted April 8, 2016

DOI: $10.3892 /$ br.2016.652

\begin{abstract}
Pulmonary lymphangioleiomyomatosis (PLAM) is a rare disease occurring frequently in women. The present study reported a case of PLAM in a 46-year-old female and reviewed the literature regarding PLAM. The female experienced dyspnea on exertion for 1 month. The chest computed tomography (CT) revealed diffuse thin-walled cystic lesions, consistent with radiological features of PLAM. CT-guided lung biopsy showed that characteristic smooth muscle cells were positive for smooth muscle actin, human melanoma black 45 and D2-40 by histology and immunohistochemistry techniques, resulting in a diagnosis of PLAM. The patient had a hysterectomy for treating uterine myoma 12 years previously. Additionally, lung diffusion function was impaired slightly, abdominal CT showed a right renal cyst and pelvic ultrasound exhibited a right oophoritic cyst. Sirolimus was administered, which improved the dyspnea and pulmonary function, and the patient required long-term follow-up. Therefore, this is a rare case of PLAM with renal cyst, oophoritic cyst and uterine myoma in a female. In conclusion, PLAM is a rare proliferative disease in the lung. Chest CT characteristically showed that the cysts were bilateral, diffuse and thin-walled. Lung biopsy may provide evidence for the diagnosis of PLAM, which was treated with sirolimus.
\end{abstract}

\section{Introduction}

Pulmonary lymphangioleiomyomatosis (PLAM) is a rare hamartomatous proliferation of the smooth muscle involved

Correspondence to: Professor Zhihong Shi and Dr Bo Zhou, Department of Respiratory and Critical Care Medicine, The First Affiliated Hospital of Xi'an Jiaotong University, 277 Yanta West Street, Xi'an, Shaanxi 710061, P.R. China

E-mail: docszh@163.com

E-mail: zb_bob@163.com

${ }^{*}$ Contributed equally

Key words: case report, pulmonary lymphangioleiomyomatosis, sirolimus, Northwestern China in blood vessels and lymphatics in the lung $(1,2)$. It extends into the pulmonary interstitium, leading to diffuse thin-walled cystic lesions and pulmonary hemorrhage, and lymph node involvement may result in chylous effusion (3). PLAM is clinically characterized by progressive dyspnea, cough, chest pain, hemoptysis and pneumothorax, leading to progressive airflow obstruction and impaired lung diffusion function, culminating in respiratory failure commonly in premenopausal women $(4,5)$. Chest radiographs show diffuse interstitial infiltrates and thin-walled cystic lesions. Pulmonary function tests usually reveal obstructive ventilation dysfunction and airflow limitation with impaired lung diffusion function. PLAM is considered to occur alone or is associated with tuberous sclerosis (6). Thus far, the pathogenesis of PLAM has not been clearly clarified and its treatment remains limited.

PLAM was first reported in China in 1986, and since then $\sim 100$ Chinese cases have been reported. However, case reports of PLAM are rare in Northwestern China. A recent retrospective study in China showed that PLAM commonly occurs in women (99.2\%); dyspnea was the most common clinical manifestation (94.6\%); the most common extrapulmonary manifestation was retroperitoneal lymph node involvement $(52.1 \%)$; the most common chest imaging manifestation was multiple different sized thin-walled cystic shadows in whole lungs (93.8\%); the most common lung biopsy method was bronchoscopic biopsy (41.0\%); in the patients with pulmonary dysfunction, obstructive ventilation dysfunction was $65.1 \%$, and diffusion dysfunction was $38.4 \%$. However, there is no effective medical therapy, and lung transplant may be the only possible treatment for the cure of patients with PLAM (7).

Regardless of the extent of the disease, it is notable that the patients are usually female, and in general are in the reproductive years. The development of PLAM is possibly associated with hormone secretion. In the present study, a woman with PLAM, renal cyst, oophoritic cyst and uterine myoma, who had a hysterectomy for treating uterine myoma 12 years previously is reported. This is a rare case report in Northwestern China.

\section{Case report}

A 46-year-old female in Northwestern China presented with exertional dyspnea, which occurred 1 month prior and 
progressed gradually. The patient did not report any fever, chills, cough, chest pain or hemoptysis. Due to worsening of symptoms, the patient visited the Department of Respiratory and Critical Care Medicine (The First Affiliated Hospital of Xi'an Jiaotong University, Xi'an, Shaanxi, China). The patient had a history of hysterectomy for treating uterine myoma 12 years previously and had no history of smoking or alcohol consumption. Physical examination appeared normal. The laboratory findings including routine tests of blood, urine and feces, liver and kidney function tests, and tumor markers detection were normal. Blood gas analysis showed slight hypoxemia, and the pulmonary function test showed a normal ventilation function with a reduced diffusing capacity of the lung for carbon monoxide (68\%). Additionally, abdominal computed tomography (CT) showed a right renal cyst, and pelvic ultrasound exhibited a right oophoritic cyst (Fig. 1).

Chest CT exhibited bilateral, diffuse and thin-walled cystic changes (Fig. 2A). Based on the chest radiographs, lung biopsy was required for a precise diagnosis. Firstly, bronchoscopy was performed and no abnormality was detected in bronchial lumen (Fig. 2B). The patient was not diagnosed by the bronchoscopic biopsy, as the size of the specimen was too small. Subsequently, CT-guided percutaneous needle-aspiration biopsy of the lung was performed for the patient (Fig. 3A). Initial microscopic examination of the lung biopsy was interpreted as fibrosis and chronic inflammation. Due to the CT findings, the pathology was questionable and the diagnosis required further assessment. Reexamination of the biopsy specimen exhibited hyperplasia of smooth muscle and a proliferation of spindle cells around small blood vessels and respiratory bronchioles (Fig. 3B). Histology and immunohistochemistry (IHC) demonstrated that characteristic smooth muscle cells were positive for smooth muscle actin (SMA), human melanoma black (HMB) 45 and D2-40 (a specific lymphatic endothelial marker) (Fig. 3B). These findings established the diagnosis of PLAM. Sirolimus was administered for the patient, which improved the dyspnea and pulmonary function temporarily. However, the patient with PLAM is in an early stage in the process of the disease and requires long-term follow-up, as there is currently no effective medical therapy for PLAM. The present study was approved by the local ethics review committees, and the patient provided informed consent.

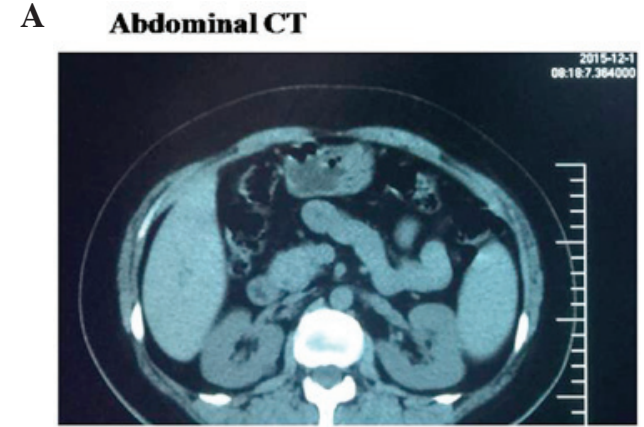

B

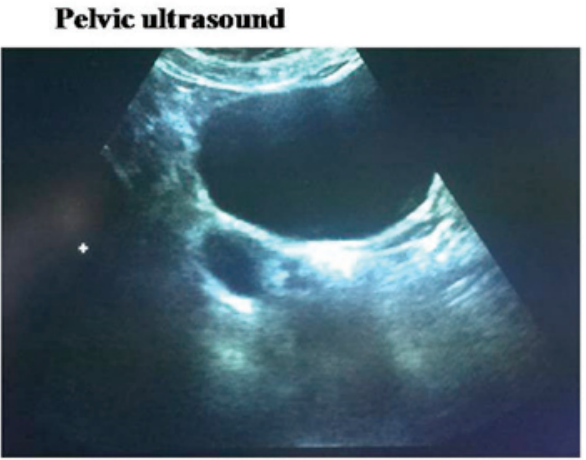

Figure 1. Assessment of a patient with pulmonary lymphangioleiomyomatosis. (A) Abdominal computed tomography (CT) and (B) pelvic ultrasound.

\section{Discussion}

PLAM is characterized by the progressive proliferation of smooth muscle in alveolar septa, pulmonary vessels and lymphatics frequently occurring in females between the ages of 17 and 50 years (8). The chest radiographs usually show that bilateral multiple pulmonary cysts. When pulmonary vessels are involved and occluded, pulmonary hemorrhage may occur in the interstitium; when the lymphatics and the thoracic duct was occluded, chylous effusion may occur. Similar results have been found in patients with tuberous sclerosis (9). PLAM is considered to be associated with tuberous sclerosis. However, in contrast to tuberous sclerosis, there is no family history, mental retardation or adenoma sebaceum in PLAM.
A

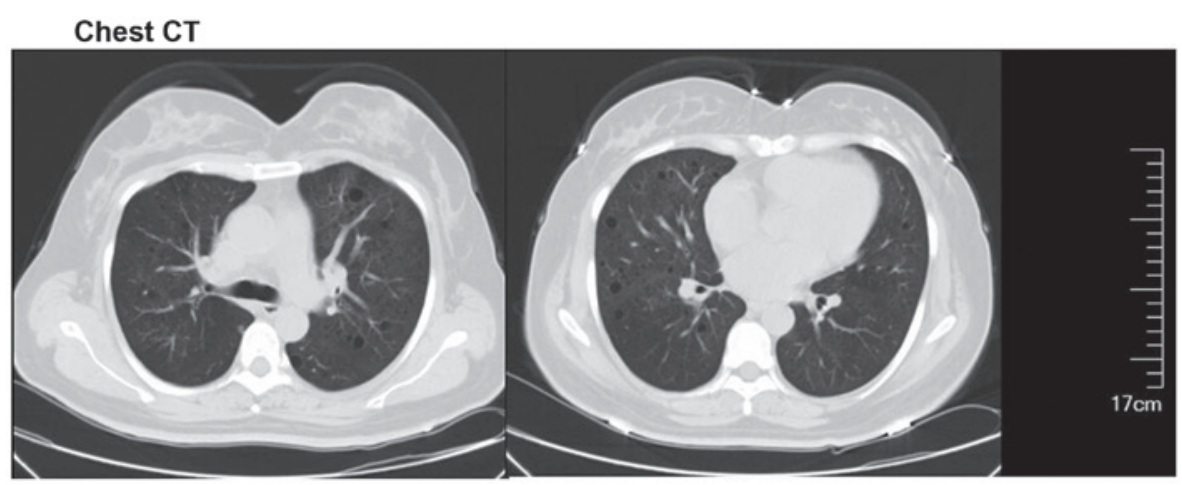

B Bronchoscopy
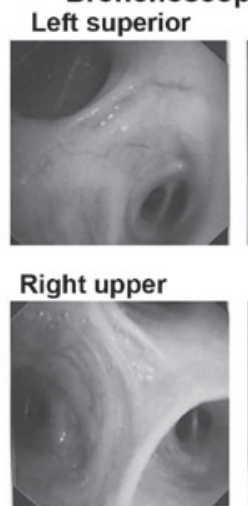

Left inferior

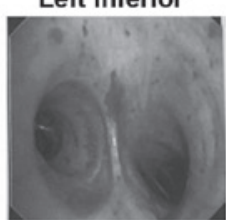

Right middle

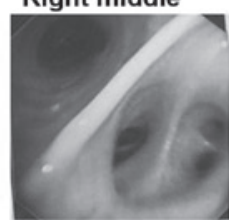

Figure 2. Assessment of a patient with pulmonary lymphangioleiomyomatosis. (A) Chest computed tomography (CT) and (B) bronchoscopy. 
A

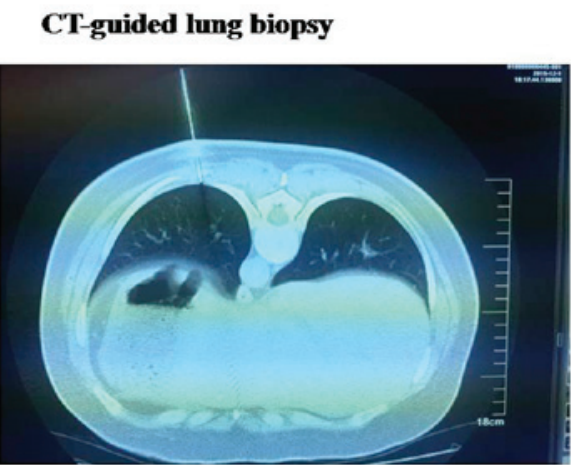

B

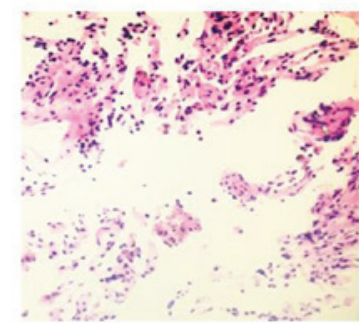

HE stain

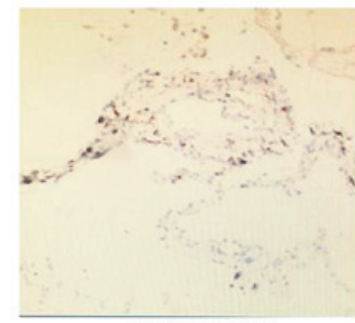

HMB45 (+)

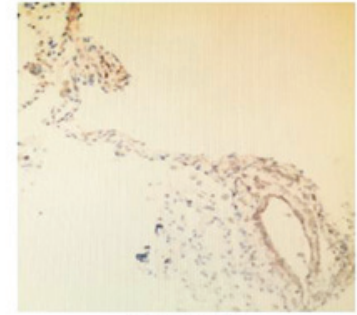

$\operatorname{SMA}(+)$

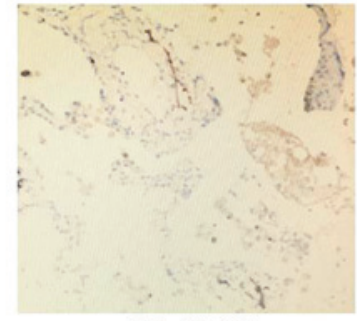

D2-40(+)
Figure 3. Assessment of a patient with pulmonary lymphangioleiomyomatosis. (A) Computed tomography (CT)-guided lung biopsy. (B) Hematoxylin and eosin staining (H\&E) of the lung biopsy samples and immunohistochemistry demonstrates positive staining for smooth muscle actin (SMA), human melanoma black 45 (HMB45) and D2-40 (magnification, x10).

The most frequent clinical manifestation worldwide is dyspnea, followed by cough, pneumothorax, chest pain, hemoptysis and chylothorax. The progressive proliferation of smooth muscle may lead to obstruction of bronchioles and pulmonary vessels, so pulmonary function tests show obstructive ventilation dysfunction and diffusion dysfunction, culminating in hypoxemia and respiratory failure. The treatment of PLAM is limited, and the prognosis remains poor. The majority of patients with PLAM may succumb to respiratory failure within 10 years (10).

In the present case, chest CT showed that the cysts were bilateral, diffuse and thin-walled. According to statistical analysis, the most common chest imaging manifestation was also multiple different sized thin-walled cystic shadows in the whole lungs. Although cysts frequently exist in various types of interstitial lung diseases, the chest radiographs of PLAM in CT are different from other interstitial lung diseases. For example, idiopathic pulmonary fibrosis is characterized by reticular abnormalities and honeycombing in the basilar and subpleural areas, while PLAM is more diffuse in the whole lungs. Furthermore, the cysts in idiopathic pulmonary fibrosis are surrounded by abnormal parenchyma, while the cysts in
PLAM are surrounded by normal tissues (11). Thus, the chest CT findings are helpful in the diagnosis of PLAM.

Currently, bronchoscopic biopsy was the most common lung biopsy method. However, sufficient specimens for the diagnosis of PLAM by bronchoscopy were not obtained, as no abnormality was identified in the bronchial lumen. This disease is rare in Northwestern China, and therefore the diagnosis procedure is unfamiliar. However, CT-guided lung biopsy showed that characteristic smooth muscle cells were positive for SMA, HMB45 and D2-40 by histology and IHC techniques, resulting in a diagnosis of PLAM. Therefore, the present study summarized the experience in clinical diagnosis and treatment of PLAM in order to improve the diagnostic accuracy rating and reduce an ignored diagnosis.

The case of PLAM in a female complicated with renal cyst, oophoritic cyst and uterine myoma that was removed by a hysterectomy 12 years previously was reported in the present study. A recent study showed that the development of PLAM is associated with hormone secretion, particularly estrogen (12). Whether renal cyst, oophoritic cyst and uterine myoma are involved in PLAM remains to be elucidated. Therefore, the patient requires long-term follow-up for observation on the process of the disease.

The results of the recent sirolimus phase III trial showed that sirolimus treatment significantly slowed the decline in the pulmonary function, and improved dyspnea and the quality of life $(13,14)$. In the present case, sirolimus treatment efficiently stabilized pulmonary function and alleviated the symptoms during the observation period. Recently, certain results have demonstrated that abnormality of the tuberous sclerosis complex gene $1 / 2$ induced LAM cell proliferation via the activation of mechanistic target of rapamycin (mTOR) (15). Thus, sirolimus, as an mTOR inhibitor, could stabilize the pulmonary function and improve dyspnea. We believe that the continuous administration of sirolimus is necessary for stabilizing the pulmonary function and improving the quality of life, and the safety and efficacy of sirolimus should be further studied in the future.

\section{Acknowledgements}

The authors appreciate the technical support and materials from the Department of Pathology (The First Affiliated Hospital of Xi'an Jiaotong University). The present study was supported by the programs from the National Natural Science Foundation of China (general program nos. 81270094 and 81500016).

\section{References}

1. Carrington CB, Cugell DW, Gaensler EA, Marks A, Redding RA, Schaaf JT and Tomasian A: Lymphangioleiomyomatosis. Physiologic-pathologic-radiologic correlations. Am Rev Respir Dis 116: 977-995, 1977.

2. Bernstein SM, Newell JD Jr, Adamczyk D, Mortenson RL, King TE Jr and Lynch DA: How common are renal angiomyolipomas in patients with pulmonary lymphangiomyomatosis? Am J Respir Crit Care Med 152: 2138-2143, 1995.

3. Corrin B, Liebow AA and Friedman PJ: Pulmonary lymphangiomyomatosis. A review. Am J Pathol 79: 348-382, 1975.

4. Hayashida M, Seyama K, Inoue Y, Fujimoto K and Kubo K; Respiratory Failure Research Group of the Japanese Ministry of Health, Labor, and Welfare: The epidemiology of lymphangioleiomyomatosis in Japan: A nationwide cross-sectional study of presenting features and prognostic factors. Respirology 12: 523-530, 2007 . 
5. Hohman DW, Noghrehkar D and Ratnayake S: Lymphangioleiomyomatosis: A review. Eur J Intern Med 19: 319-324, 2008.

6. Valensi QJ: Pulmonary lymphangiomyoma, a probable forme frust of tuberous sclerosis. A case report and survey of the literature. Am Rev Respir Dis 108: 1411-1415, 1973.

7. Zhang L, Liang Y and Zhong X and Liu J: Literature review of clinical and pathological features of pulmonary lymphangioleiomyomatosis for 130 cases in China in the last thirty years. Chin Gen Pract 3: 329-334, 2015.

8. Silverstein EF, Ellis K, Wolff M and Jaretzki A III: Pulmonary lymphangiomyomatosis. Am J Roentgenol Radium Ther Nucl Med 120: 832-850, 1974

9. Sato T, Seyama K, Fujii H, Maruyama H, Setoguchi Y, Iwakami S, Fukuchi Y and Hino O: Mutation analysis of the TSC1 and TSC2 genes in Japanese patients with pulmonary lymphangioleiomyomatosis. J Hum Genet 47: 20-28, 2002.

10. Taylor JR, Ryu J, Colby TV and Raffin TA: Lymphangioleiomyomatosis. Clinical course in 32 patients. N Engl J Med 323: 1254-1260, 1990.
11. Kitaichi $\mathrm{M}$, Nishimura $\mathrm{K}$, Itoh $\mathrm{H}$ and Izumi $\mathrm{T}$ : Pulmonary lymphangioleiomyomatosis: A report of 46 patients including a clinicopathologic study of prognostic factors. Am J Respir Crit Care Med 151: 527-533, 1995

12. Numata T, Araya J, Mikami J, Hara H, Harada T, Takahashi H, Nakayama K and Kuwano K: A case of pulmonary lymphangioleiomyomatosis complicated with uterine and retroperitoneal tumors. Respir Med Case Rep 15: 71-76, 2015.

13. McCormack FX, Inoue Y, Moss J, Singer LG, Strange C, Nakata K, Barker AF, Chapman JT, Brantly ML, Stocks JM, et al; National Institutes of Health Rare Lung Diseases Consortium; MILES Trial Group: Efficacy and safety of sirolimus in lymphangioleiomyomatosis. N Engl J Med 364: 1595-1606, 2011.

14. Carsillo T, Astrinidis A and Henske EP: Mutations in the tuberous sclerosis complex gene TSC 2 are a cause of sporadic pulmonary lymphangioleiomyomatosis. Proc Natl Acad Sci USA 97: 6085-6090, 2000.

15. Yang Q and Guan KL: Expanding mTOR signaling. Cell Res 17: 666-681, 2007. 